\title{
EVALUACIÓN DE DIVERSOS PATRONES HETERÓTICOS EN LA FORMACIÓN DE HÍBRIDOS DE MAÍZ PARA EL BAJÍO MEXICANO
}

\author{
Humberto de León ${ }^{1}$, Ernestor Ramírez ${ }^{2}$, Gaspar Martínez ${ }^{1}$, Arnoldo Oyervides ${ }^{2}$, Alfredo de la Rosa ${ }^{2}$
}

\begin{abstract}
RESUMEN
Evaluación de diversos patrones heteróticos en la formación de híbridos de maíz para el bajío mexicano. El presente trabajo se realizó con los objetivos de evaluar y caracterizar diversos patrones heteróticos como una estrategia de mejoramiento en la formación de híbridos para el Bajío Mexicano, a través del comportamiento de las cruzas formadas; la estimación del tipo de acción génica dentro de cada patrón heterótico y la identificación de híbridos sobresalientes para la región del Bajío Mexicano. El material consistió de tres grupos heteróticos: Tropical por Bajío (TxB); Enano por Normal (ExN) y Precoz por Tardío (PxT), con 20 híbridos cada uno y dos testigos comerciales recomendados para la región. Los híbridos se formaron mediante un diseño de apareamiento anidado, en el cual cada uno de cinco machos se cruzó con cuatro hembras. Esto permitió considerarlos como familias de hermanos completos y utilizarlos en la caracterización genética de los grupos o patrones heteróticos. Los híbridos se evaluaron para rendimiento de mazorca a $15,5 \%$ de humedad, días a floración masculina y femenina y altura de planta y mazorca. Las cruzas se evaluaron en el ciclo primavera-verano de 1995, en dos localidades representativas del Bajío Mexicano. Los resultados mostraron que el patrón PxT produjo los híbridos con mejor varianza genética y con las mejores características agronómicas. Las dificultades intrínsecas de asincronía entre los progenitores para la formación de los híbridos indicaron al patrón ExN como la mejor estrategia de formación de híbridos para el Bajío Mexicano.
\end{abstract}

\begin{abstract}
Evaluation of heterotic patterns in developing maize hybrids for middle-altitude regions of Mexico. This research was carried out to evaluate and characterize several heterotic patterns as a breeding strategy for developing hybrids for the Bajio region in Mexico. It was based on the performance of the crosses made, in the estimation of the type of gene action within each heterotic pattern, and the proportion of high yielding hybrids for intermediate or middle-altitude regions of Mexico. The genetic material evaluated included three heterotic groups: Tropical x Bajio (TxB); Dwarf x Normal (DxN), and Early x Late (ExL), with 20 hybrids each one and two commercial controls recommended for the region. Hybrids were developed using a nested mating design, where each of five males was crossed with four females. Hybrids were considered as full-sibs, and then, utilized for the genetic characterization of the groups or heterotic patterns. Data were recorded for ear yield at 15,5\% moisture content, days to anthesis, days to silking and plant height. Hybrid evaluation was conducted in two representative locations of middle-altitude (1100 to 1900 masl) regions during the spring-summer season of 1995 at Celaya, Gto.and Sandia el Grande, N.L. Results showed that the ExL pattern produced hybrids having the best agronomic traits and the highest genetic variance. Due to the intrinsic asynchrony difficulties it was concluded that the DxL pattern was the best strategy for developing hybrids for the middlealtitude regions of Mexico.
\end{abstract}

\footnotetext{
1 Profesor-Investigador, Departamento de Fitomejoramiento, UAAAN. Saltillo, Coahuila, México. E mail: gmartin@uaaan.mx

2 Departamento de Fitomejoramiento, UAAAN.
} 


\section{INTRODUCCIÓN}

El cruzamiento de materiales con características contrastantes incluyendo adaptabilidad, proporciona altos niveles de heterosis. La clasificación o agrupamiento de líneas de maíz y la programación de cruzas a partir de patrones heteróticos específicos para una cierta región, permite una mayor seguridad en la formación de híbridos sobresalientes en ese ambiente. Cuando se está interesado en elegir un patrón heterótico para la formación de híbridos en una determinada región, lo más recomendable es hacerlo mediante la evaluación de los diferentes patrones desarrollados para ese ambiente y con base en ello seleccionar el mejor.

Por lo anterior, se realizó el presente trabajo en el Instituto Mexicano del Maíz "Dr. Mario E. Castro Gil”, con los objetivos de evaluar y caracterizar diversos patrones heteróticos como una estrategia de mejoramiento en la formación de híbridos para El Bajío Mexicano, a través del comportamiento de las cruzas formadas; la estimación del tipo de acción génica dentro de cada patrón heterótico y la proporción de híbridos sobresalientes para la región de El Bajío Mexicano. Todo esto atendiendo, entre otras argumentaciones, a la acertada indicación de Córdova y Mickelson (1995), de que la apropiada elección del germoplasma constituye la mitad del éxito, en un programa de hibridación; así mismo, mencionan que ello implica tener el conocimiento del patrón heterótico a que pertenece; así como a la valiosa aportación de Robinson, Comstock y Harvey, (1949) quienes afirman que las variaciones en los caracteres cuantitativos, son el principal interés del mejorador, ya que la naturaleza de la acción génica es un factor importante en la evaluación de varios procedimientos de mejoramiento.

\section{MATERIALES Y MÉTODOS}

El material lo constituyó tres grupos heteróticos: Tropical por Bajío (TxB); Enano por Normal (ExN) y Precoz por Tardío (PxT), con 20 híbridos cada uno y dos testigos comerciales recomendados para la región. Los híbridos se formaron mediante un diseño de apareamiento anidado (Comstock y Robinson, 1948), en el cual cada uno de cinco machos se cruzó con cuatro hembras. Esto permitió considerarlos como familias de hermanos completos y utilizarlos en la caracterización genética de los grupos o patrones heteróticos.

Los híbridos se evaluaron para rendimiento de mazorca al 15,5\% de humedad, días a floración masculina $\mathrm{y}$ femenina y altura de planta y mazorca.
La formación de las cruzas experimentales se efectuó en el ciclo de invierno 1994-1995, en la localidad de Tepalcingo, Mor. Estas cruzas se evaluaron en el ciclo primavera-verano de 1995, en dos localidades representativas del Bajío Mexicano que son: Celaya, Gto. situada a $20^{\circ} 32^{\prime}$ de latitud Norte y $100^{\circ} 49^{\prime}$ de longitud Oeste, con una altura de $1754 \mathrm{msnm}$; con una temperatura media anual de $20,6^{\circ} \mathrm{C}$ y una precipitación pluvial anual de 597,3 mm. Sandia el Grande, N.L. situada a $24^{\circ} 49^{\prime}$ de latitud Norte y $100^{\circ} 04^{\prime}$ de latitud Oeste, con una altura de $1654 \mathrm{msnm}$; con una temperatura media anual de $18,6^{\circ} \mathrm{C}$ y una precipitación pluvial anual de $365,0 \mathrm{~mm}$.

La siembra se efectuó para cada una de las localidades, de manera independiente de acuerdo a las fechas de siembra establecidas regionalmente. La parcela experimental en las dos localidades fue de un surco de 4,41 m de largo, por 0,80 $\mathrm{m}$ de ancho, con 21 plantas por surco. La siembra del experimento se llevó a cabo en forma manual, depositando dos semillas por golpe, para posteriormente aclarear a una planta así asegurar al número óptimo de plantas. El desarrollo del cultivo fue bajo condiciones de riego, con la aplicación plaguicidas cuando fue necesario.

La fórmula de fertilización aplicada (N-P-K), fue 180-90-00, la cual se distribuyó en dos partes; la primera al momento de la siembra (90-90-00), y la segunda en el primer cultivo. El número de riegos aplicados fue variable, en función de los requerimientos específicos de cada localidad.

Los patrones heteróticos evaluados explican satisfactoriamente el comportamiento superior de los híbridos y existirá diferencia entre ellos, cuyo comportamiento está determinado por el tipo de acción génica no aditivo.

En lo referente al diseño, fue un factorial con dos repeticiones, las fuentes de variación no estimadas se acumularon en error experimental.

También se estimó la prepotencia de cada uno de los 15 machos incluidos en el presente estudio, con el objeto de seleccionar dentro de cada patrón, la cruza simple de más altos efectos de Aptitud Combinatoria General; la fórmula empleada fue:

$$
P P i j=\frac{\sum x i j}{n}
$$

Donde:

ppij = Prepotencia o ACG de cada una de las cruzas simples (machos) progenitoras de los híbridos dobles evaluados. 
Cuadro 1. Análisis de varianza combinado general a través de patrones heteróticos (grupos) y ambientes.

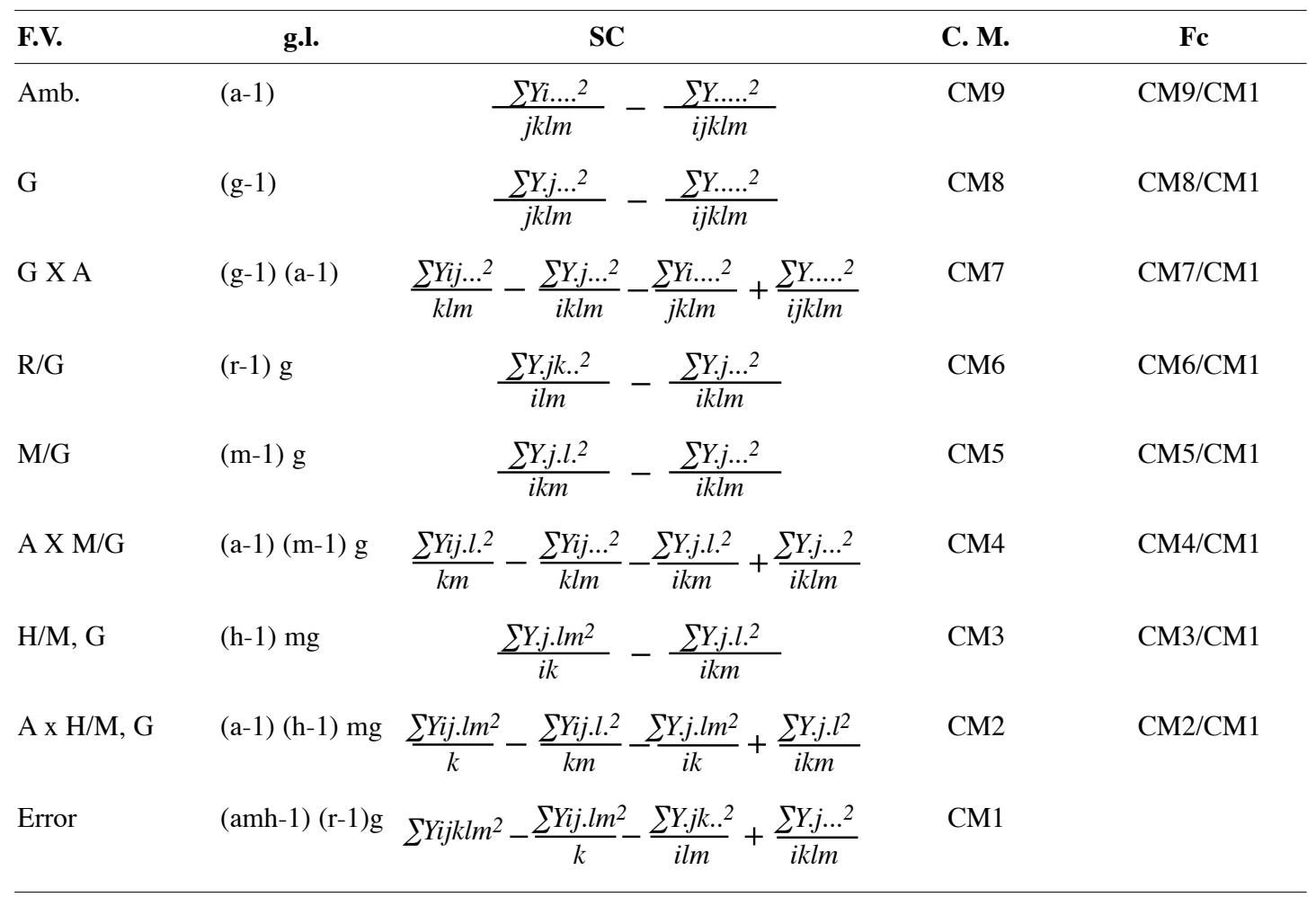

Cuadro 2. Análisis de varianza combinado de ambientes del Diseño I de Carolina del Norte.

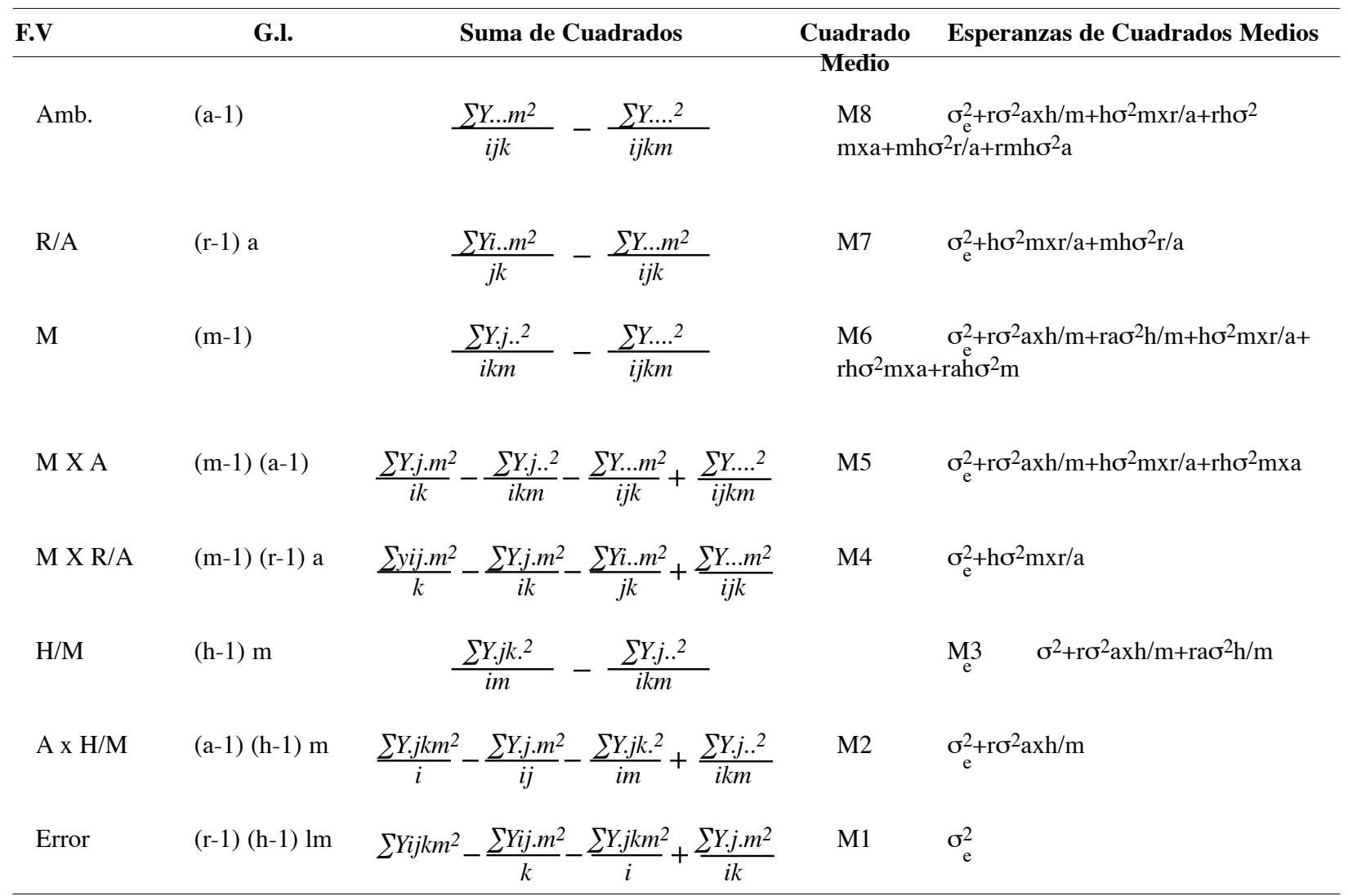


$\mathrm{S}(\mathrm{xij})=$ Suma de los híbridos donde intervienen cada progenitor a través de ambientes.

$\mathrm{n} \quad=$ Número de híbridos donde interviene el progenitor en evaluación.

por último, con el fin de estimar la varianza genética dentro de cada patrón, se calculó su coeficiente mediante la siguiente fórmula:

$$
C V G=\frac{\sqrt{\sigma^{2} m+\sigma^{2} h / m}}{\bar{X}} \times 100
$$

Donde:

CVG = Coeficiente de variación genética

$\sigma^{2} \mathrm{~m} \quad=$ Varianza de machos

$\sigma^{2} \mathrm{~h} / \mathrm{m}=$ Varianza de hembras dentro de machos

= Media del carácter

\section{RESULTADOS Y DISCUSIÓN}

En el Cuadro 4 se resumen los cuadrados medios del análisis combinado general, del que podemos resaltar lo siguiente: en cuanto a los ambientes de evaluación, se detecta una clara diferencia entre ellos, por la alta significancia arrojada por los caracteres en estudio. La siguiente fuente es la de grupos y se refiere a los diferentes patrones heteróticos empleados, observándose una alta significancia en todos los caracteres evaluados, de lo que se infiere que la información genética que cada patrón aporta a sus descendientes, tiende a ser diferente al comportamiento de otro grupo. Semejante tendencia la muestran los machos dentro de grupos y hembras dentro de machos y grupos, indicando con ello que es factible realizar selección, ya que estadísticamente son diferentes sus comportamientos. En atención a esta información, se detectaron los machos con efectos más favorables de ACG, así como las hembras

Cuadro 3. Análisis de Varianza individual del Diseño I de Carolina del Norte.

\begin{tabular}{|c|c|c|c|c|c|c|c|}
\hline F.V & g.l & & & SC & & C. M. & E. C. $\mathbf{M}$ \\
\hline $\mathrm{R}$ & $(\mathrm{r}-1)$ & $\frac{\sum Y i .^{2}}{j k}$ & - & $\frac{\sum Y \ldots{ }^{2}}{i j k}$ & & M4 & \\
\hline M & $(\mathrm{m}-1)$ & $\frac{\sum Y . j^{2}}{i k}$ & - & $\frac{\sum Y \ldots{ }^{2}}{i j k}$ & & M3 & $\sigma_{\mathrm{e}}^{2}+\mathrm{r} \sigma^{2}+\mathrm{rh} \sigma^{2} \mathrm{~m}$ \\
\hline $\mathrm{H} / \mathrm{M}$ & $(h-1) \mathrm{m}$ & $\frac{\sum Y . j k^{2}}{i}$ & - & $\frac{\sum Y . j^{2}}{i k}$ & & M2 & $\sigma_{\mathrm{e}}^{2}+\mathrm{r} \sigma^{2} \mathrm{~h} / \mathrm{m}$ \\
\hline Error & $(\mathrm{r}-1)(\mathrm{mh}-1)$ & $\sum Y i j k^{2}-$ & $\frac{\sum Y . j k^{2}}{i}$ & $-\frac{\sum Y i . .^{2}}{j k}$ & $+\frac{\sum Y \ldots{ }^{2}}{i j k}$ & M1 & $\sigma_{\mathrm{e}}^{2}$ \\
\hline
\end{tabular}

Cuadro 4. Cuadrados medios y su significancia del análisis de varianza combinado de tres grupos heteróticos, evaluados a través de dos ambientes. Celaya, Gto. y Sandia el Grande, N.L., 1995.

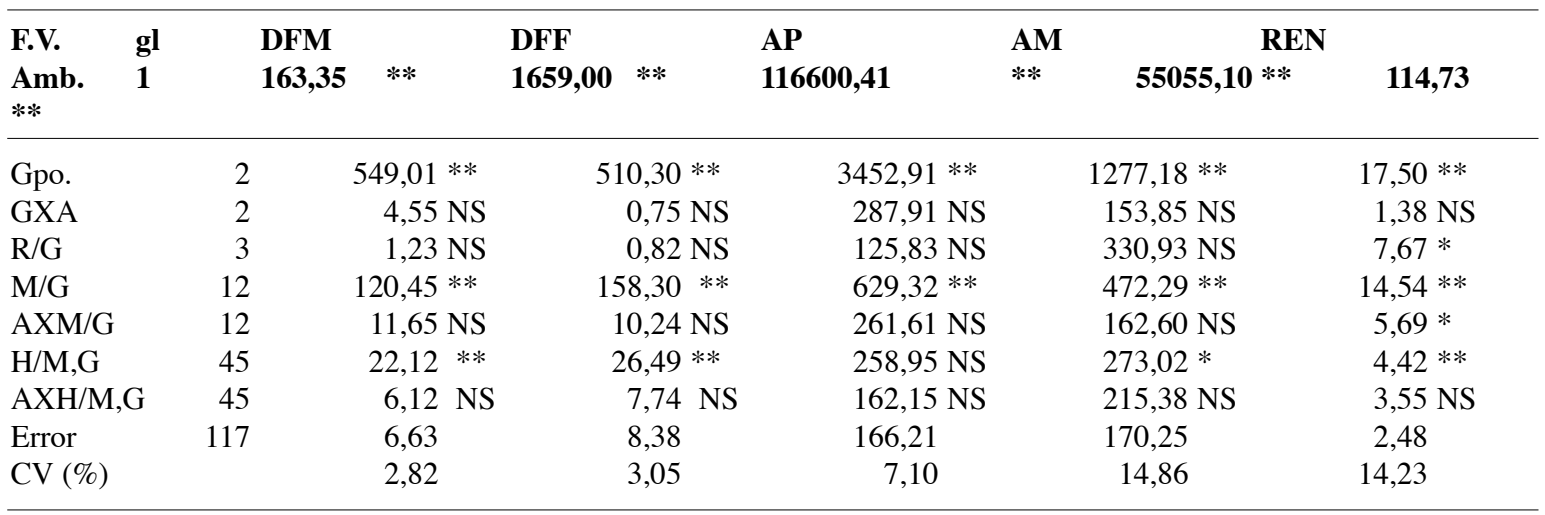

*,**,Significativo al 0,05 y 0,01 de probabilidad, respectivamente. NS, No significativo.

DFM, DFF= Días a floración masculina y femenina; AP y AMZ= Altura de planta y mazorca; REN= Rendimiento de mazorca al $15,5 \%$ de humedad. 
Cuadro 5. Componentes de la variación genética de cinco caracteres en tres patrones heteróticos de maíz evaluados en dos localidades representativas de El Bajío Mexicano, 1995.

\begin{tabular}{lrrrrr}
\hline Componente & DFM & DFF & AP & AMZ & REN \\
\hline Bajíi x Tropical & & & & & \\
s2A & 7,61 & 7,65 & 59,91 & $-34,47$ & 1,82 \\
s2D & $-2,54$ & $-3,10$ & 2,16 & 216,15 & $-4,45$ \\
CVG(\%) & 1,92 & 1,82 & 2,91 & 7,30 & 5,90 \\
Enano x Normal & & & & & \\
s2A & 43,56 & 48,66 & 82,29 & $-6,33$ & 3,06 \\
s2D & $-23,60$ & $-23,90$ & $-198,90$ & $-54,92$ & $-2,98$ \\
CVG(\%) & 4,27 & 4,42 & 2,57 & 0 & 7,73 \\
Precoz x Tardío & & & & & \\
s2A & 18,44 & 58,83 & 61,38 & 229,84 & 0,95 \\
s2D & 4,53 & $-31,88$ & 284,03 & $-177,30$ & 4,23 \\
CVG(\%) & 3,64 & 5,03 & 5,61 & 9,69 & 11,72 \\
\hline
\end{tabular}

s2A = Varianza Aditiva; s2D=Varianza de Dominancia; CVG=Coeficiente de Variación Genética.

DFM, DFF= Días a floración masculina y femenina; AP y AMZ= Altura de planta y mazorca; REN= Rendimiento de mazorca al $15.5 \%$ de humedad.

Cuadro 6. Medias de cinco caracteres en tres patrones heteróticos de maíz evaluados en dos localidades representativas de El Bajío Mexicano, 1995.

\begin{tabular}{lccccc}
\hline Patrón & DFM & DFF & AP & AMZ & REN \\
\hline BxT & $92,4 \mathrm{a}^{1}$ & $95,7 \mathrm{a}$ & $188,9 \mathrm{a}$ & $92,3 \mathrm{a}$ & $11,4 \mathrm{a}$ \\
ExN & $93,2 \mathrm{a}$ & $96,8^{\mathrm{a}}$ & $176,1 \mathrm{~b}$ & $84,5 \mathrm{~b}$ & $11,3 \mathrm{a}$ \\
PxT & $88,3 \mathrm{~b}$ & $92,0 \mathrm{~b}$ & $179,8 \mathrm{~b}$ & $86,7 \mathrm{~b}$ & $10,6 \mathrm{~b}$ \\
\hline
\end{tabular}

BxT= BajíoxTrópico; ExN= EnanoxNormal; PxT= Precoz $\mathrm{x}$ Tardío.

DFM, DFF= Días a floración masculina y femenina; AP y $\mathrm{AMZ}=$ Altura de planta y mazorca; REN= Rendimiento de mazorca al $15,5 \%$ de humedad.

1 Medias con la misma letra en cada columna, son estadísticamente iguales al 0,05 de probabilidad.

dentro de machos con comportamiento agronómico igual o superior a los testigos comerciales.

Los siguientes cuadros muestran que la varianza aditiva fue mayor, en general, a su contraparte no aditiva en las cinco características estudiadas, en los tres grupos heteróticos (Cuadro 5). Lo que implica que para seguir obteniendo expresiones heteróticas satisfactorias, no se requiere de la conducción de un esquema de selección recíproca recurrente entre las poblaciones contrastantes en cada patrón heterótico. El patrón PxT produjo los híbridos con mejor varianza genética (Cuadro 5) y con las mejores características agronómicas; en cambio, los patrones ExN y BxT mostraron mejor rendimiento (Cuadro 6). La formación de híbridos con el patrón PxT ofrece dificultades prácticas difíciles de superar en un programa de producción a gran escala de variedades híbridas, derivadas de la asincronía obvia entre los progenitores. El patrón ExN no presenta sólo tal dificultad, sino que facilita la polinización natural de los progenitores, ya que por lo regular se usa al progenitor enano como hembra. El patrón ExN se comportó de manera similar al PxT en altura de planta y mazorca (Cuadro 6), por lo que puede considerarse en términos prácticos, como la mejor estrategia de formación de híbridos para El Bajío Mexicano.

El patrón PxT produjo los híbridos con mejor varianza genética y con las mejores características agronómicas; sin embargo, las dificultades intrínsecas de asincronía entre los progenitores para la formación de los híbridos, conduce a la consideración del patrón ExN como la mejor estrategia de formación de híbridos para El Bajío Mexicano.

\section{LITERATURA CITADA}

CÓRDOBA, O.H.; MICKELSON, R.H. 1995. CIMMYT maize program internally managed. External review of strategies and methodologies. El Batán, México . p 6.

COMSTOCK, E. R.; ROBINSON, F.H. 1948. Biometrics 4:254-266.

ROBINSON,F.H.; COMSTOCK, R. E.; HARVEY. B. H. 1949. Estimates of heretability and the degree of dominance in corn. Agron. J. 41:353-359. 